\title{
Enhancement of Doppler Unambiguity for Chirp-Sequence Modulated TDM-MIMO Radars
}

Fabian Roos, Jonathan Bechter, Nils Appenrodt, Jürgen Dickmann, and Christian Waldschmidt

(C) 2018 IEEE. Personal use of this material is permitted. Permission from IEEE must be obtained for all other uses, in any current or future media, including reprinting/republishing this material for advertising or promotional purposes, creating new collective works, for resale or redistribution to servers or lists, or reuse of any copyrighted component of this work in other works. 


\title{
Enhancement of Doppler Unambiguity for Chirp-Sequence Modulated TDM-MIMO Radars
}

\author{
Fabian Roos*, Jonathan Bechter*, Nils Appenrodt ${ }^{\dagger}$, Jürgen Dickmann ${ }^{\dagger}$, and Christian Waldschmidt* \\ ${ }^{*}$ Institute of Microwave Engineering, Ulm University, 89081 Ulm, Germany \\ ${ }^{\dagger}$ Daimler AG, Group Research and Advanced Engineering, 89081 Ulm, Germany \\ Email: fabian.roos@uni-ulm.de
}

\begin{abstract}
Current automotive radar sensors enhance the angular resolution using a multiple-input multiple-output approach. The often applied time-division multiplexing scheme has the drawback of a reduced unambiguous Doppler velocity proportional to the number of transmitters. In this paper, a signal processing scheme is proposed to regain the same unambiguous Doppler as in the single-input multiple-output case with only one transmit antenna. Simulation and measurement results are shown to prove that the signal processing leads to an enhanced unambiguous Doppler velocity estimation.
\end{abstract}

\section{INTRODUCTION}

Driver assistance systems require radar sensors that offer high-resolution as well as large unambiguous range, velocity, and angle estimations. As the angular resolution is linked to the aperture size of the antenna array, a common procedure is to use a multiple-input multiple-output (MIMO) configuration to increase the aperture. Possible choices to generate the required orthogonal signals are time-division (TDM), frequencydivision (FDM), or code-division (CDM) multiplexing [1], [2].

The often used TDM scheme is shown in Fig. 1 for two transmitters, which are active in an alternating way. Hence, the chirp repetition time of the same transmit antenna is enlarged by the number of different transmit antennae $M$. This is a major drawback as the maximum unambiguously detectable radial velocity

$$
v_{\max }= \pm \frac{c}{2 f_{\mathrm{c}}} f_{\mathrm{D}, \max }= \pm \frac{c}{2 f_{\mathrm{c}}} \frac{1}{2 M T_{\mathrm{r}}}
$$

depends on the centre frequency $f_{c}$, the number of different transmitters $M$, and the chirp repetition time $T_{\mathrm{r}}$ between two consecutive chirps. In this example the unambiguous Doppler spectrum is halved. Additionally, the time difference between the different active transmit antennae introduces a phase error which depends on the radial velocity of the respective target [3].

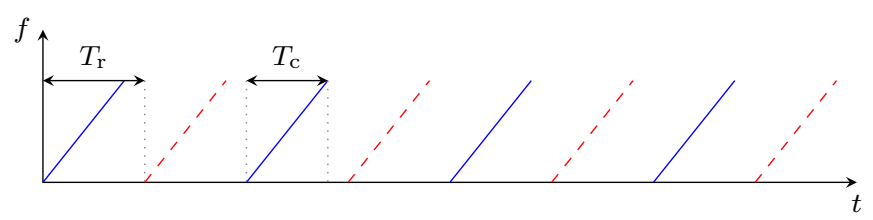

Fig. 1. Two transmitter are active alternatively in the TDM-MIMO scheme. The Doppler frequency is sampled with the chirp repetition time $T_{\mathrm{r}}$, which can be interpreted as the sampling frequency of the Doppler.
To regain a larger maximum unambiguous velocity the authors of [4] alter the classic chirp-sequence modulation format [5] by transmitting the consecutive frequency ramps with different starting frequencies. This enables an interlaced evaluation and therewith a larger unambiguous velocity. Another possibility is to transmit consecutive frequency ramps with different chirp repetition times. The resulting different unambiguous domain can be evaluated with the Chinese remainder theorem (CRT) [6].

To compensate the second drawback, the motion-induced phase error, different procedures are known in literature. One possibility is to create the virtual aperture such that it contains one position with two virtual elements from both transmit antennae. As the phase information should be identically at this position, this can be used to compensate the phase error [3]. In [7] a signal processing scheme is presented which does not rely on a so-called overlapping element.

In this paper a signal processing approach is proposed which is based on the last mentioned processing scheme to enhance the unambiguous Doppler velocity. As an advantage, the classic chirp-sequence modulation can be used and the chirp repetition times do not need to be altered. Hence, this approach could be used with available state-of-the-art radar sensors to regain the same unambiguous Doppler velocity as in the single-input multiple-output TDM case.

\section{Phase ERror Compensation}

In the case when only one transmit antenna is active, the measured phase difference of the received signal between different receiving elements is only dependent on the angle of incidence. In the TDM-MIMO case the switching time between the different active transmit antennae introduces an additional Doppler velocity dependent phase error [3]. This phase error needs to be compensated for a correct angle estimation.

Usually, the Doppler velocity is estimated with the samples $s[k]$ of the same range cell across consecutive frequency chirps using the discrete Fourier transform (DFT)

$$
S\left(f_{\mathrm{D}}\right)=\sum_{k} s[k] \mathrm{e}^{-\mathrm{i} 2 \pi f_{\mathrm{D}} k T_{\mathrm{r}}},
$$

where $T_{\mathrm{r}}$ is the ramp repetition interval [5]. To overcome the motion-induced phase error the DFT is altered according to [7]. The key idea is that the signals received from different 

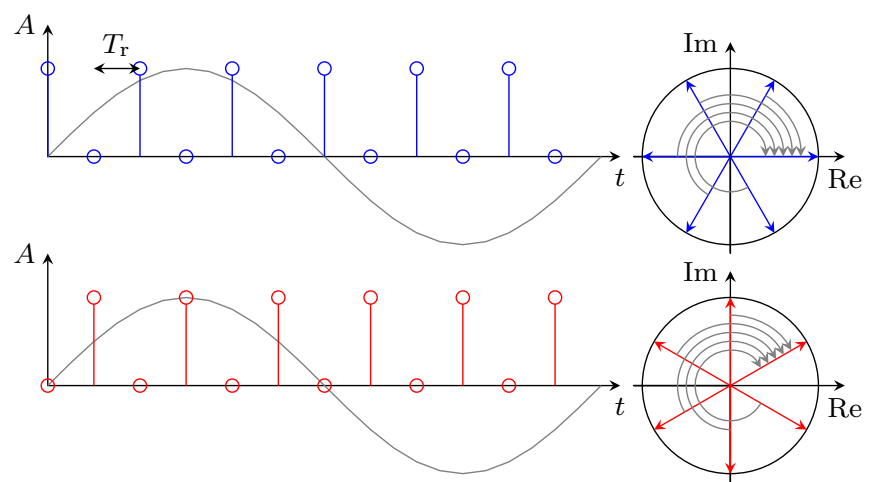

Fig. 2. With two transmit antennae operating at the TDM-MIMO mode the Doppler frequency $(-)$ is sampled at different times. Only the available samples are shown as complex vectors in the unit circle on the right. If the missing samples in between are not taken into consideration, this results in a motion-induced phase error as the DFT rotates all vectors to the first one.

transmit antennae are measured with a time difference as depicted in Fig. 2. In this example there is a difference by one sample, i.e. frequency ramp, because of the two transmit antennae. On the right side the unit circle is depicted with complex vectors for the available frequency ramps after range compression. The DFT rotates all vectors to the initial vector resulting in a peak in spectrum at the corresponding frequency. If the two resulting phases are compared there is an error if the missing samples are not considered. By taking the missing samples into account this phase error can be compensated by altering the DFT for the two antennae to

$$
S_{\mathrm{Tx}_{1}}\left(f_{\mathrm{D}}\right)=\sum_{k} s_{\mathrm{Tx}_{1}}[k] \mathrm{e}^{-\mathrm{i} 2 \pi f_{\mathrm{D}}(2 k) T_{\mathrm{r}}},
$$

with $s_{\mathrm{Tx}_{1}}$ being a received signal transmitted from antenna 1 and to

$$
S_{\mathrm{Tx}_{2}}\left(f_{\mathrm{D}}\right)=\sum_{k} s_{\mathrm{Tx}_{2}}[k] \mathrm{e}^{-\mathrm{i} 2 \pi f_{\mathrm{D}}(2 k+1) T_{\mathrm{r}}}
$$

for the second transmit antenna. In general, if $M$ transmit antennae are present the DFT for the $m \in[0, M-1]$ antennae is

$$
S_{\mathrm{Tx}_{m}}\left(f_{\mathrm{D}}\right)=\sum_{k} s_{\mathrm{Tx}_{m}}[k] \mathrm{e}^{-\mathrm{i} 2 \pi f_{\mathrm{D}}(M k+m) T_{\mathrm{r}}} .
$$

As pointed out in [7] this is equivalent to an interlaced zeropadding in Doppler dimension.

\section{Enhancement of DopPler Ambiguity}

For a target with a Doppler velocity in the unambiguous domain the peaks at $f_{\mathrm{D}}<f_{\mathrm{D}, \max }$ can be described by (3) and (4) for two transmitters. Considering the interlaced zeropadding processing spectral repetitions occur at the positions shown in Fig. 3.

If a target has a Doppler velocity larger than the unambiguous domain $\tilde{f}_{\mathrm{D}}>f_{\mathrm{D}, \max }$, the spectral repetition $\tilde{f}_{\mathrm{D}}-2 f_{\mathrm{D} \text {, max }}$ will be considered as the valid target. This leads for the first antenna to

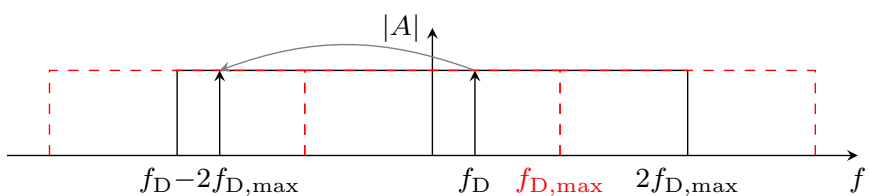

(a) Spectral peak and spectral repetition for $f_{\mathrm{D}}<f_{\mathrm{D} \text {, max }}$.

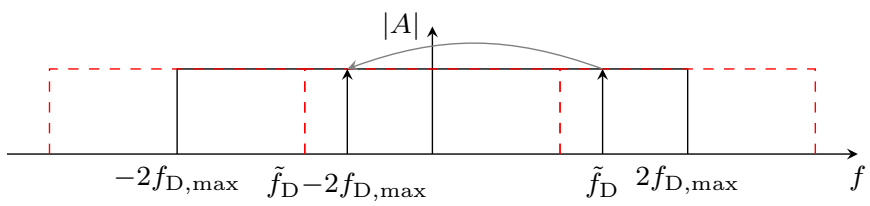

(b) Spectral peak and spectral repetition for $\tilde{f}_{\mathrm{D}}>f_{\mathrm{D} \text {,max }}$.

Fig. 3. Due to the TDM-MIMO operation with two transmitters and the interlaced zero-padding the original Doppler spectrum $\left[-f_{\mathrm{D}, \max }, f_{\mathrm{D}, \max }\right]$ $(---)$ is enlarged with spectral repetitions to $\left[-2 f_{\mathrm{D}, \max }, 2 f_{\mathrm{D}, \max }\right](-)$.

$$
\begin{aligned}
S_{\mathrm{Tx}_{1}}\left(\tilde{f}_{\mathrm{D}}-2 f_{\mathrm{D}, \max }\right) & \stackrel{(3)}{=} \sum_{k} s_{\mathrm{Tx}_{1}}[k] \mathrm{e}^{-\mathrm{i} 2 \pi f_{\mathrm{D}}(2 k) T_{\mathrm{r}}} \cdot \underbrace{\mathrm{e}^{+\mathrm{i} 2 \pi k}}_{=1} \\
& =S_{\mathrm{Tx}_{1}}\left(\tilde{f}_{\mathrm{D}}\right)
\end{aligned}
$$

and for the second transmit antenna to

$$
\begin{aligned}
S_{\mathrm{Tx}_{2}}\left(\tilde{f}_{\mathrm{D}}-2 f_{\mathrm{D}, \max }\right) \stackrel{(4)}{=} & \sum_{k} s_{\mathrm{Tx}_{2}}[k] \mathrm{e}^{-\mathrm{i} 2 \pi f_{\mathrm{D}}(2 k+1) T_{\mathrm{r}}} \\
& \cdot \underbrace{\mathrm{e}^{+\mathrm{i} 2 \pi k+\mathrm{i} \pi}}_{=\mathrm{e}^{\mathrm{i} \pi}} \\
= & S_{\mathrm{Tx}_{2}}\left(\tilde{f}_{\mathrm{D}}\right) \cdot \mathrm{e}^{\mathrm{i} \pi} .
\end{aligned}
$$

As can be seen from (7) there is a phase offset of $\pm \pi$ present, if the detected velocity is in the range of $\left[-2 f_{\mathrm{D}, \max },-f_{\mathrm{D}, \max }\right]$ or $\left[f_{\mathrm{D}, \max }, 2 f_{\mathrm{D}, \max }\right]$, i.e. the actual velocity is in the first spectral repetition.

In the case of $M$ transmitters the phase offset for the $m \in[0, M-1]$ antennae for the $\xi$ th spectral repetition is

$$
\begin{aligned}
S_{\mathrm{Tx}_{\mathrm{m}}} & \left(\tilde{f}_{\mathrm{D}}-\frac{\xi m}{M} f_{\mathrm{D}, \max }\right) \\
& \stackrel{(5)}{=} \sum_{k} s_{\mathrm{Tx}_{\mathrm{m}}}[k] \mathrm{e}^{-\mathrm{i} 2 \pi\left(\tilde{f}_{\mathrm{D}}-\frac{\xi m}{M} f_{\mathrm{D}, \max }\right)(M k+m) T_{\mathrm{r}}} \\
= & S_{\mathrm{Tx}_{\mathrm{m}}}\left(\tilde{f}_{\mathrm{D}}\right) \mathrm{e}^{+\mathrm{i} 2 \pi \frac{\xi m}{M}} .
\end{aligned}
$$

This relationship is shown for an example of $M=4$ transmitters in Fig. 4. The phase trend of the detected target $f_{\mathrm{D}}$ in the unambiguous frequency range is shown for the virtual aperture elements of different transmitters. For a velocity which is unambiguously detected, i.e. $\xi=0(\uparrow)$, there are no phase offsets. If a target $(\uparrow)$ with a velocity in the first spectral repetition $\xi=1$ is present, there are phase offsets between the virtual antenna elements of different transmitters. This phase offset equals

$$
\varphi_{1, m}=\mathrm{e}^{+\mathrm{i} 2 \pi \frac{\xi m}{M}}
$$

for the transition from the first to the $m$ th transmitter and, e.g. for the second repetition $\left({ }^{\uparrow}\right), \xi=2$ can be calculated. Hence, 

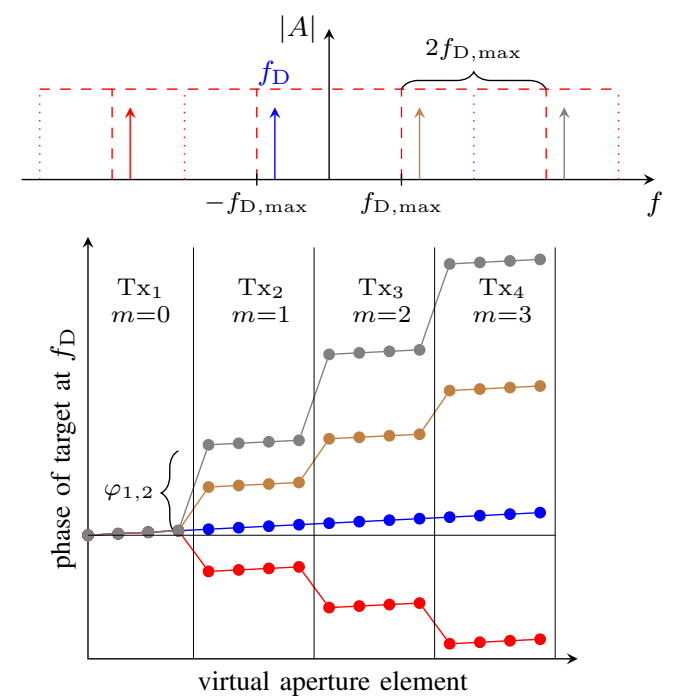

Fig. 4. Phase trends of targets with different radial velocities according to (9).

the observed phase offset is used to estimate $\xi$ and therefore the actual velocity of a target is determined.

It should be noted, that with an increasing number of transmitters the phase offset (9) decreases and therefore the detection is dependent on a reliable phase estimation.

For this simulation the same parameters are used as in the measurement presented later on. The simulated target in Fig. 5 moves with a velocity one spectral repetition beyond the unambiguous limit. As two transmitters are present, the phase offset is $\pi$ as derived in (7) in this case. For the direction of arrival estimation, this results in a minimum at the exact azimuth angle (----). Considering the phase offset and determining the correct spectral repetition using (9) leads to an angle estimation (-.-.) which corresponds to the undisturbed single-input multiple-output (SIMO) case (-) but with a much narrower peak.

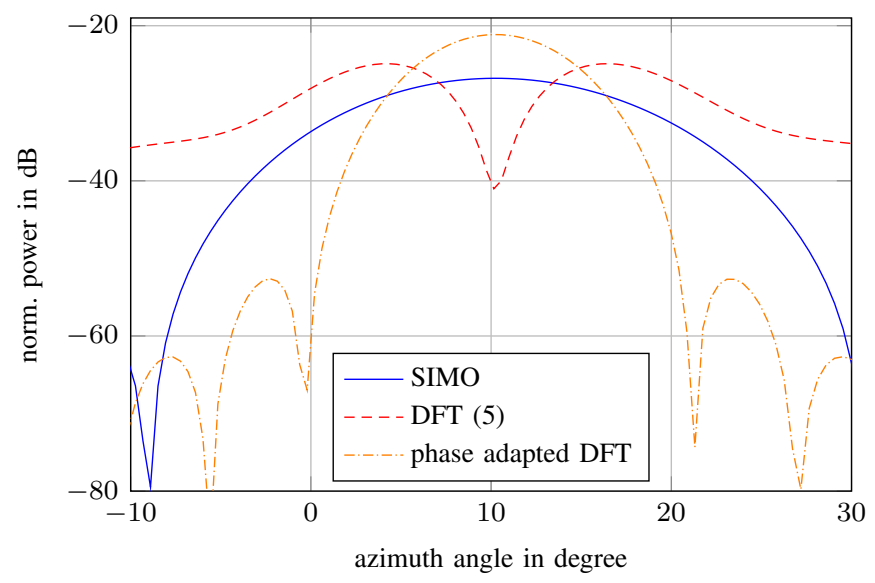

Fig. 5. Simulation of a moving target with a velocity larger than the unambiguous domain. This phase offset leads to a deformed spectrum (-- - ) if it is not detected and corrected (-.-). SIMO (- ${ }^{-}$is used as reference.
TABLE I

SPECIFICATIONS OF RADAR PARAMETERS

\begin{tabular}{lr} 
Parameter & Value \\
\hline carrier frequency $f_{\mathrm{c}}$ & $76.41 \mathrm{GHz}$ \\
bandwidth $B$ & $594 \mathrm{MHz}$ \\
chirp duration $T_{\mathrm{c}}$ & $20.480 \mu \mathrm{s}$ \\
chirp repetition time $T_{\mathrm{r}}$ & $27.015 \mu \mathrm{s}$ \\
sampling frequency $f_{\mathrm{s}}$ & $25 \mathrm{MHz}$ \\
number of chirps $L$ & 256 \\
$v_{\max , \text { MIMO }}$ & $\pm 18.15 \frac{\mathrm{m}}{\mathrm{s}}$ \\
$v_{\max , \text { SIMO }}$ & $\pm 36.01 \frac{\mathrm{m}}{\mathrm{s}}$
\end{tabular}

\section{Measurement Results}

For verification an experimental radar sensor is mounted at the front of a vehicle which drives in a static environment. The parameters of the used modulation format are listed in Tab. I. As the TDM-MIMO mode is activated, the total number of 256 frequency chirps is transmitted from both antennae. The resulting virtual aperture of the uniform linear array (ULA) includes an overlapping element for verification purpose.

The static environment is a multi-target one, i.e. a metal fence with it's poles at the right side of the street is detected as numerous strong point targets. In the resulting range-Doppler evaluation shown in Fig. 6 all ten physical receive channels are combined. As the velocity is larger than the unambiguous velocity, part of the static environment is visible as spectral repetitions with large positive velocities. To illustrate the repetitions the range-Doppler plot is extended beyond the unambiguous domain. For the discrimination between a target and noise, an ordered-statistic constant false alarm rate (OSCFAR) algorithm and a peak search algorithm is applied.

A strong detection $(\times)$ is selected and the phase trend according to Fig. 4 is shown in Fig. 7. With the DFT processing (5) a phase jump of $\varphi_{1,2}=-\pi$ is detected $(-*)$.

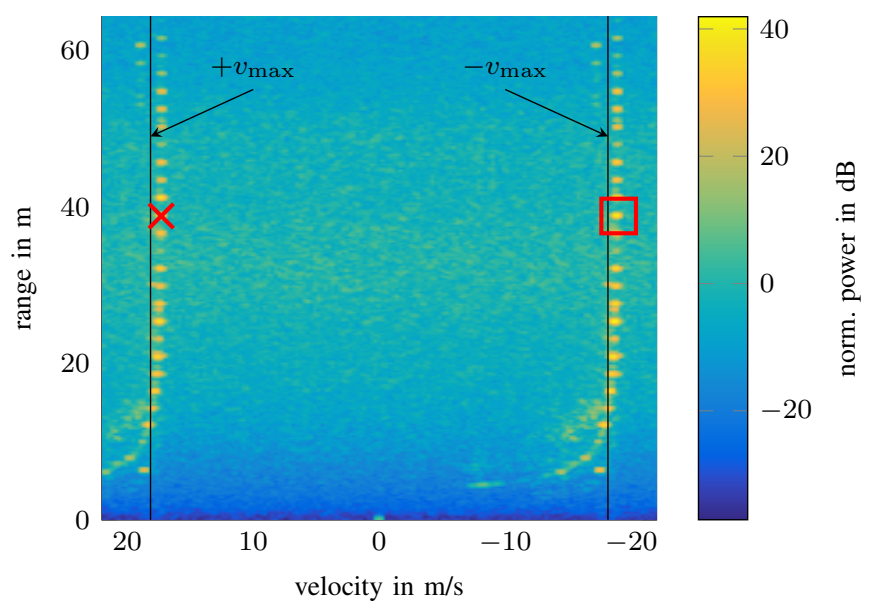

Fig. 6. Measurement of a stationary scene from an approaching radar sensor mounted at a vehicle. As the velocity is too large some targets are detected with a positive velocity due to the spectral repetition. A prominent target $(X)$ and the corresponding spectral repetition $(\square)$ beyond the unambiguous domain are selected for angle estimation. 


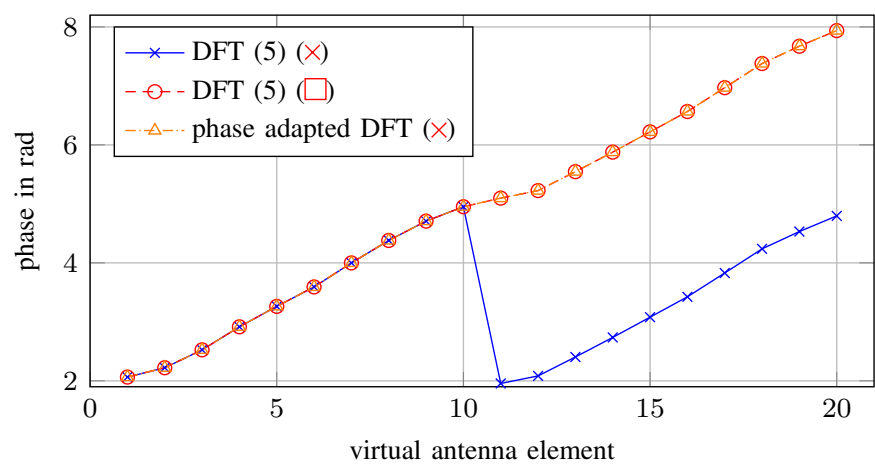

Fig. 7. Phase trend of the two selected targets $(\times, \square)$ according to Fig. 4 .

Using the relation (9), this results in $\xi=-1$ and with (8) the actual Doppler frequency

$$
f_{\mathrm{D}, \text { estimated }}=f_{\mathrm{D}, \text { real }}-\frac{-1 \cdot 1}{2} f_{\mathrm{D}, \max }
$$

is calculated $(\square)$. Hence, the phase adapted DFT ( $-\triangle \cdots)$ has the same phase trend as the peak using the spectral repetition $\left(--^{-}\right)$.

In Fig. 8 the angle estimation is shown for the two selected peaks of Fig. 6. As the peak in the unambiguous domain $(\times)$ is a spectral repetition, the already mentioned phase offset leads to a deformed angle estimation if not corrected (----). To show that the spectral repetition $(\square)$ corresponds to the real velocity, the angle estimation is performed (-.-.) and compared to the SIMO case (-).

Fig. 9 summarises and compares the proposed signal processing to the approach using an overlapping element. For the selected peak $(\times)$ the phase trend is evaluated and a detected offset is corrected. This leads to a direction of arrival estimation (-.-.) with the same result as the overlapping element approach (-). As the phase offset in the overlapping element approach is determined using two single phase measurements, the results are inferior compared to the adapted DFT process-

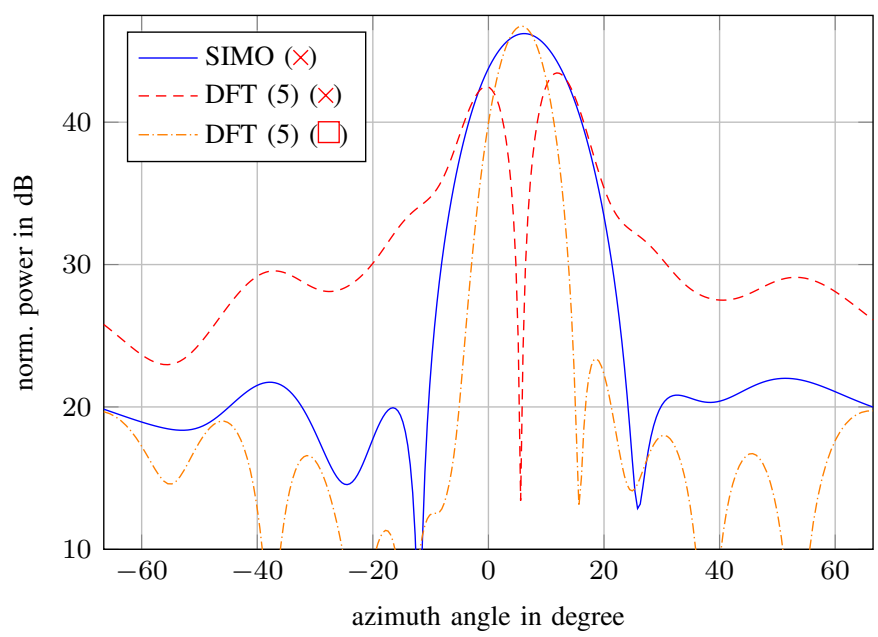

Fig. 8. Azimuth estimation for the peaks of Fig. 6 compared to SIMO case.

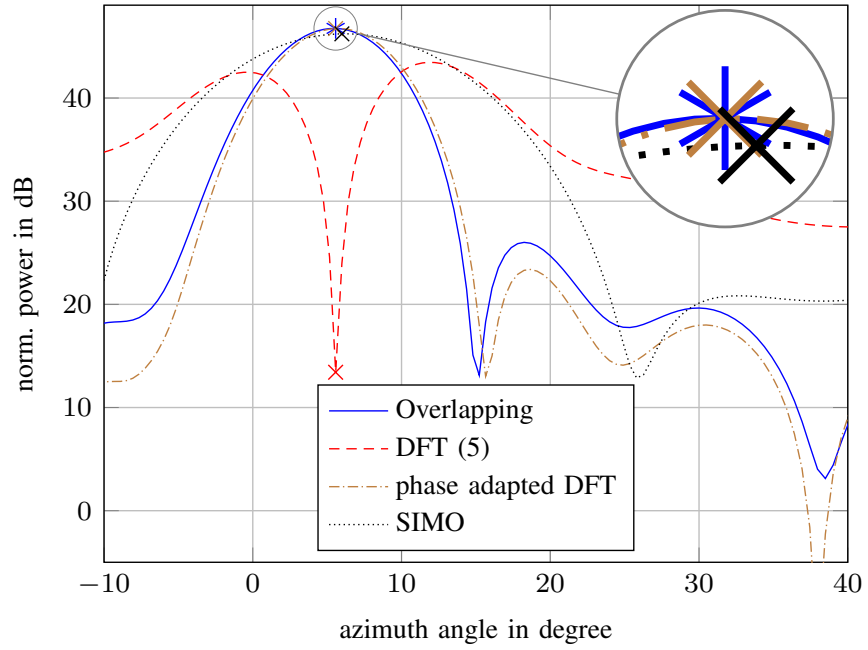

Fig. 9. Angle estimation for the selected target $(X)$ inside the unambiguous Doppler velocity of Fig. 6 .

ing. It can be seen that the SIMO estimation ( $\quad$ - ) yields the same azimuth angle.

\section{CONClusion}

Different signal processing approaches are known in literature to enhance the unambiguous Doppler velocity measured with a radar sensor. In contrast to the Chinese remainder theorem or the interlaced chirp-sequence approach the modulation format does not need to be altered with the processing scheme presented in this paper. It is solely based on the phase evaluation of the virtual aperture. Hence, this signal processing can be applied in existing radar sensors without the requirement of an overlapping element in the virtual aperture. It is possible to regain the same unambiguous Doppler velocity as in the corresponding SIMO case for only one transmit antenna. Simulation and measurement results are presented to validate the processing scheme.

\section{REFERENCES}

[1] J. J. M. de Wit, W. L. van Rossum, and A. J. de Jong, "Orthogonal Waveforms for FMCW MIMO Radar," in IEEE RadarCon (RADAR), May 2011, pp. 686-691.

[2] H. Sun, F. Brigui, and M. Lesturgie, "Analysis and Comparison of MIMO Radar Waveforms," in International Radar Conference, Oct. 2014, pp. $1-$ 6.

[3] C. M. Schmid, R. Feger, C. Pfeffer, and A. Stelzer, "Motion Compensation and Efficient Array Design for TDMA FMCW MIMO Radar Systems," in 6th European Conference on Antennas and Propagation (EUCAP), Mar. 2012, pp. 1746-1750.

[4] K. Thurn, D. Shmakov, G. Li, S. Max, M.-M. Meinecke, and M. Vossiek, "Concept and Implementation of a PLL-Controlled Interlaced Chirp Sequence Radar for Optimized Range-Doppler Measurements," IEEE Transactions on Microwave Theory and Techniques, vol. 64, no. 10, pp. 3280-3289, Oct. 2016.

[5] V. Winkler, "Range Doppler Detection for automotive FMCW Radars," in Proceedings of the 4th European Radar Conference (EuRAD), Oct. 2007, pp. 166-169.

[6] H. Rohling, "Resolution of Range and Doppler Ambiguities in Pulse Radar Systems," in Proceedings of Digital Signal Processing, 1987.

[7] J. Bechter, F. Roos, and C. Waldschmidt, "Compensation of MotionInduced Phase Errors in TDM MIMO Radars," IEEE Microwave and Wireless Components Letters, vol. 27, no. 12, pp. 1164-1166, Dec. 2017. 